\title{
New molecular target for the phylogenetic identification of Leptospira species directly from clinical samples: an alternative gene to $16 \mathrm{~S}$ rRNA
}

\author{
Rafael Guillermo Villarreal Julio[1], Piedad Agudelo-Flórez ${ }^{[1]}$, \\ Juan Álvaro López ${ }^{[2]}$ and Ronald Guillermo Peláez Sánchez ${ }^{[1]}$
}

\author{
[1]. Basic Science Research Group, Graduates School - CES University. Medellín, Antioquia, Colombia. \\ [2]. Microbiology School, Primary Immunodeficiencies Group, University of Antioquia. Medellín, Antioquia, Colombia.
}

\begin{abstract}
Introduction: Phylogenetic analysis of the $16 \mathrm{~S}$ ribosomal gene initial region is used to identify Leptospira isolates at the species level from clinical samples. Unfortunately, this method cannot differentiate between some intermediates and saprophytic species. Methods: We used comparative genomic analysis between 35 Leptospira species to find new molecular targets for Leptospira species identification. Results: We proposed the use of the rpoC gene, encoding the DNA-directed RNA polymerase $\beta$-subunit, for identifying 35 Leptospira species. Conclusions: The rpoC gene can be a molecular target to identify the main species of the Leptospira genus directly from clinical samples.
\end{abstract}

Keywords: Leptospira. Species. Phylogenetic. Typing. rpoC.

Leptospirosis is a globally distributed zoonotic disease caused by pathogenic bacteria of the Leptospira genus ${ }^{1}$. Currently, a phylogenetic analysis of the $16 \mathrm{~S}$ ribosomal gene is used to identify new isolates at the species level from clinical samples ${ }^{2}$. Most studies use the initial gene region, $45 \%$ of which contains gene polymorphisms. Unfortunately, with this gene region, it is not possible to differentiate between L. biflexa/L. wolbachii, L. meyeri/L. macculloughii/L. levettii/L. yanagawae, L. licerasiae/L. saintgironsiae/L. neocaledonica, L. brenneri/L. harrisiae, and L. venezuelensis/L. haakeii/L. hartskeerlii/L. wolffii species ${ }^{3}$. Therefore, it is necessary to amplify and sequence the complete gene (1500 base pairs, approximately), which greatly decreases PCR sensitivity in the diagnosis and identification of clinical samples. Additionally, deduced phylogeny of the complete gene cannot be used to discriminate between L. meyeri and L. yanagawae ${ }^{4}$. Therefore, the discovery of new molecular targets for diagnosis and species identification from clinical samples is necessary. We present

\footnotetext{
Corresponding author: Ronald Guillermo Peláez Sánchez.

e-mail: rpelaezp@ces.edu.co

(D) 0000-0002-2815-9844

Received 5 July 2019

Accepted 6 September 2019
}

a comparative genomic analysis between 35 Leptospira species, with the aim of finding new molecular targets for the identification of Leptospira species.

\section{Phylogenetic analysis of $16 \mathrm{~S}$ ribosomal gene}

The reference sequences of 35 Leptospira species were downloaded from the NCBI database. These species represent pathogenic, intermediate, and saprophytic subgroups. The evolutionary history was inferred using the Neighbor-Joining method. The tree topologies were evaluated by using a bootstrap test, and the values were obtained after 1000 replicates of the dataset. The evolutionary distances were computed using the Kimura 2-parameter method. A total of 331 positions were obtained in the final dataset. Evolutionary analyses were conducted with the Molecular Evolutionary Genetics Analysis (MEGA)-V7 softwares

\section{Orthologous proteins detection and percent protein sequence identity}

The proteomes were used for the identification of orthologous proteins. The OrthoMCL (for the identification of orthologous groups in eukaryotic genomes) and OrthoVenn (a web server for genome-wide comparison and annotation of orthologous clusters across multiple species) bioinformatics tools were used to cluster 
proteins into orthologous groups based on the identity of their sequences $^{6,7}$, and the InParanoid algorithm (orthologous groups with in-paralogous genes) was used to identify pairwise orthologous proteins $^{8}$. The percentage of sequence similarity between Leptospira species was determined using the Rapid Annotation Using Subsystem Technology (RAS) server ${ }^{9}$. The percentage of sequence similarity between proteins was initially determined between 19 Leptospira species because the RAST web server only allowed for analyses by groups of 10 species. Subsequently, the presence of orthologous proteins was verified in the remaining 16 species.

\section{Choice and analysis of candidate proteins}

The proteins that were conserved among the 35 Leptospira species were selected for manual extraction of their coding sequences (CDS) from their genomes. These CDS were aligned using the MEGA-V7 software to identify conserved regions among CDS for the design of genus-specific primers and polymorphic regions that allow for the differentiation of species.

\section{Design and verification of primers}

The primers were designed using the Primers 3 bioinformatics program. Dimers, heterodimers, and hairpins were evaluated with the Oligo Analyzer software, and the cross-amplification test was performed with Primer-BLAST NCBI bioinformatics tool.

\section{Phylogenetic analysis of rpoC gene}

The evolutionary history was inferred using the NeighborJoining method. The percentage of replicate trees in which the associated taxa clustered together in the bootstrap test (1000 replicates) was determined. The evolutionary distances were computed using the Kimura 2-parameter method. There were a total of 353 positions in the final dataset. Evolutionary analyses were conducted in MEGA-V7.

\section{Experimental verification of the PCR-rpoC gene}

A $353 \mathrm{bp}$ fragment from the $r p o C$ gene was amplified by polymerase chain reaction (PCR). The reagent concentrations used for PCR standardization were as follows: primers (forward, 5'-CAAGGGGTTCATATCAACGATAA-3'; reverse, 5'-GTTCCGGCAGGGATCATGTGACC-3'), $0.4 \mu \mathrm{M}$; dNTPs, 0.2 $\mathrm{mM}$; buffer, $1 \times$; $\mathrm{MgCl} 2,1.5 \mathrm{mM}$; Taq polymerase, 1 unit/reaction; and DNA, $200 \mathrm{ng} / \mu \mathrm{L}$. The final volume for each reaction was 25 $\mu \mathrm{L}$. The PCR was performed in a Perkin Elmer 9700 thermocycler. The thermal cycling profile was as follows: one initial denaturation cycle at $94^{\circ} \mathrm{C}$ for 5 minutes, followed by 35 cycles at $94^{\circ} \mathrm{C}$ for 35 seconds, $58^{\circ} \mathrm{C}$ for 45 seconds, $72^{\circ} \mathrm{C}$ for 1 minute, and a final cycle at the extension temperature of $72^{\circ} \mathrm{C}$ for 5 minutes.

\section{Leptospira detection from reference strains and clinical samples}

Five pathogenic (L. interrogans, L. noguchii, L. kirschnerii, $L$. santarosai, and L. borgpetersenii), two intermediates (L. fainei, and $L$. inadai), and one saprophytic species (L. biflexa) were used for the PCR-rpoC standardization. Six samples from humans, rodents, and monkeys naturally infected with Leptospira were used to verify the usefulness of the PCR in detecting Leptospira from clinical samples (the samples were donated by the leptospirosis research group of the CES University). Additionally, the detection limit of PCR assay for the $r p o C$ gene was evaluated using serial dilutions (1/10), starting with $100 \mathrm{ng}$ of DNA from the $L$. interrogans reference strain.

\section{rpoC gene sequencing in humans, rodents and monkey's samples}

To confirm the species identification from the phylogenetic analysis, a $353 \mathrm{bp}$ fragment of the $r p o C$ gene from each sample was amplified and purified using a gel extraction kit (Qiagen). The concentration and purity were determined using a Nanodrop, whereas integrity was assessed by electrophoresis with a $1 \%$ agarose gel. All amplification products were sent to the Macrogen ${ }^{\circledR}$ company (Seoul, Korea) for sequencing. For each sample, both forward and reverse sequences were used to generate a consensus sequence using Mega7. The phylogenetic analysis of the rpoC gene was performed as previously described.

Phylogenetic analysis of the partial $16 \mathrm{~S}$ ribosomal gene resulted in the correct separation of the Leptospira genus from the genetically closest genus (Leptonema illini), an adequate separation of the pathogenic, intermediate, and saprophytic subgroups, and the ability to differentiate $20 / 35$ species currently described ${ }^{10}$ with branch supports between $16 \%$ and $100 \%$. Unfortunately, with this gene region, it was not possible to differentiate between $L$. biflexa/L. wolbachii, L. meyeri/L. macculloughii/L. levettii/L. yanagawae, L. licerasiae/ L. saintgironsiae/L. neocaledonica, L. brenneri/L. harrisiae, and L. venezuelensis/L. haakeii/L. hartskeerlii/L. wolffi species (Figure 1).

Successful extraction of 35 reference genomes from the NCBI database corresponding to different Leptospira species was achieved (AE010300.2, AOHC00000000.2, AHOC00000000.2, AKWY00000000.2, АHMT00000000.2, GCA000332555.2, CP000348.1, CP006694.1, AHMP00000000.2, GCA_003429505.1, NZ_NPDU $00000000.1, \mathrm{NZ}$ _NPEF $00000000.1, \mathrm{NZ}$ NPDS00000000.1, AKWX00000000.2, AHMM00000000.2, AKWZ00000000.2, AHMO00000000.2, AHOO00000000.2, GCA_002150035.1, NZ_NPDZ00000000.1, NZ_ NPEA0̄0000000.1, NZ_NPDL00000000.1,NZ_NPEG00000000.1, NZ_NPDR00000000.1, AOGY00000000.2, CP000786.1, AKXE00000000.1, AOGZ00000000.2, AOGW00000000.2, AOGX00000000.2,NZ_NPEK00000000.1, NZ_NPDQ00000000.1, NZ_NPDW00000000.1, NZ_NPDM00000000.1, NZ RQHW00000000.1). Additionally, the reference sequences of the $r p o C$ and $16 \mathrm{~S}$ ribosomal genes were extracted manually from these genomes for the subsequent phylogenetic analyses.

A query of orthologous proteins between the 35 Leptospira species revealed 1650 genus-specific proteins [Supplementary data (Figure 2A)]. An amino acid identity analysis among the different species show a high genetic diversity between different Leptospira species. The saprophytic species were the most conserved, while the pathogenic and intermediate species showed a high genomic plasticity [Supplementary data (Figure 2B)]. Additionally, the most polymorphic proteins, but with conserved regions, were selected for primer design and subsequent phylogenetic analysis from its coding regions.

A manual evaluation of orthologous proteins conserved in the genus was made with the following inclusion parameters for the 


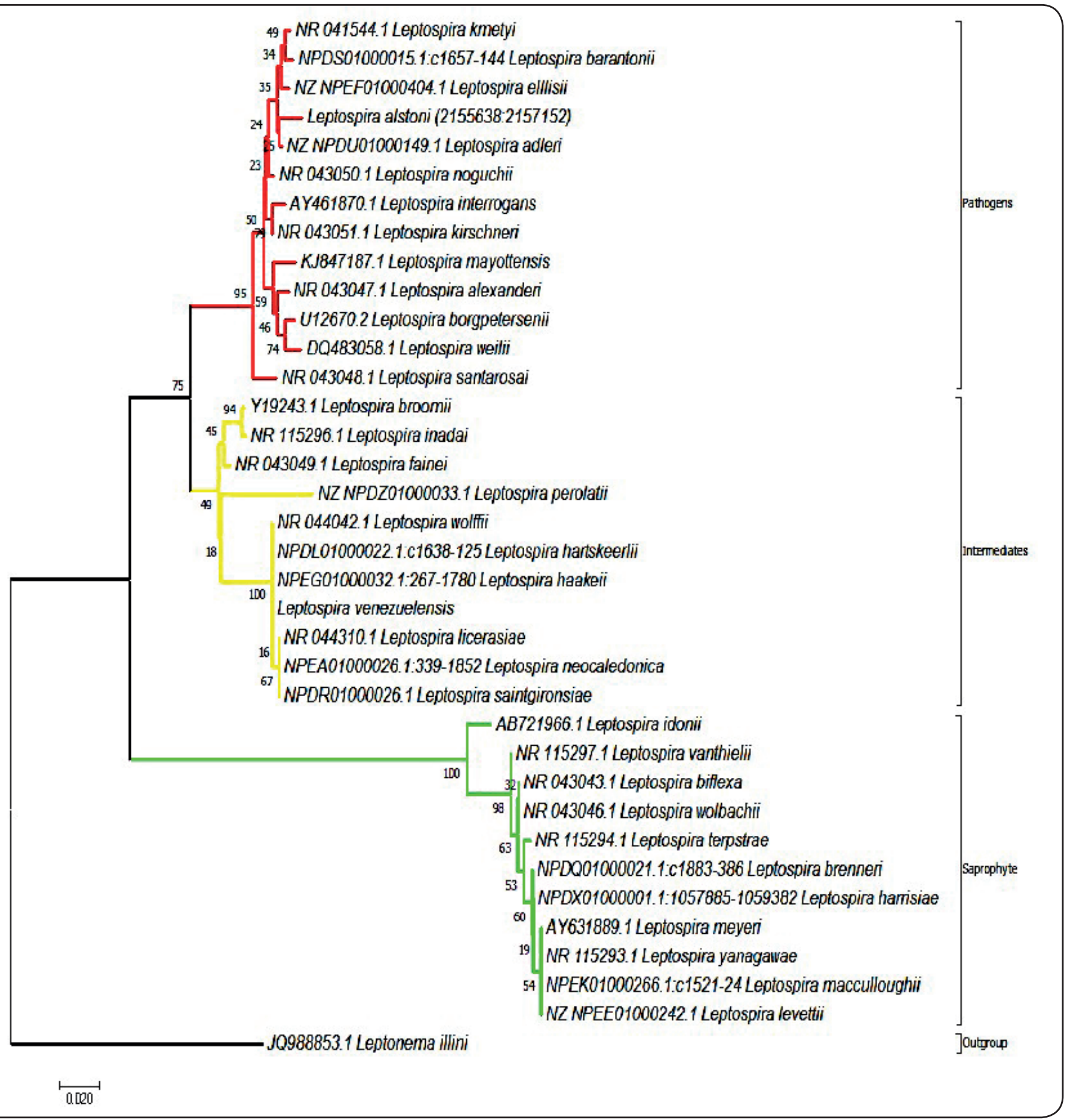

FIGURE 1: Identification of species belonging to the Leptospira genus using phylogenetic analysis of the 16S rRNA gene. The phylogenetic reconstructions for the 16S ribosomal gene are shown. The red, yellow, and green lines represent pathogenic, intermediate, and saprophytic species, respectively. The figure shows that the $16 \mathrm{~S}$ ribosomal gene cannot be used to differentiate between L. biflexa/L. wolbachii, L. meyeri/L. macculloughii/L. levettii/L. yanagawae, L. licerasiae/L. saintgironsiae/L. neocaledonica, and L. venezuelensis/L. haakeii/L. hartskeerlii/L. wolffii.

proteins: (1) the protein must be present in all species, (2) the gene must have conserved regions for genus-specific primer design, and (3) the genetic polymorphisms should allow for the differentiation of the 35 Leptospira species. Interestingly, high variability in nucleotide sequences and low variability in amino acid sequences were found; these findings could be related to genetic differences between the three species-subgroups and may have an important role in virulence. From these conserved proteins, we selected the DNA-directed RNA polymerase subunit beta protein ( $r p o C$ gene) because this protein was conserved in all species of the Leptospira genus, and its polymorphism permits genus, subgroups, and species identification. Additionally, this protein contains conserved regions that allow for the design of gender-specific primers. 


\section{Design and verification of primers}

The $r p o C-F$ and $r p o C-R$ primers were designed in two conserved regions of the rpo $C$ gene using the Primers 3 bioinformatics tool. The in silico amplification product was $353 \mathrm{bp}$. Dimers $(4.01 \mathrm{kcal} / \mathrm{mol})$, heterodimers $(4.41 \mathrm{kcal} / \mathrm{mol})$, and hairpins $(0.87 \mathrm{kcal} / \mathrm{mol})$ were evaluated with the Oligo Analyzer software. A cross-amplification test was performed with the Primer-BLAST NCBI bioinformatics tool. No cross-amplification of sizes close to $353 \mathrm{bp}$ were obtained. Only one possible amplification of 476 bp was detected in Runella sp, Bacteroidetes and Spirosoma radiotolerans, but the primers did not have a $100 \%$ alignment with these species; therefore, an amplification would not be obtained.

\section{Phylogenetic analysis of rpoC gene}

The rpoC gene sequences of 35 Leptospira species were obtained from the NCBI database. To delimit the phylogenetic analysis, the rpoC-F and $r p o C-R$ primers were used. A common $353 \mathrm{bp}$ fragment for each species was used for the phylogenetic analysis. As a result of the sequence alignment, we found that 58.07\% (205 nucleotides) and 41.92\% (148 nucleotides) were conserved and variable, respectively. A phylogenetic analysis of the rpoC gene resulted in the correct separation of the Leptospira genus, an adequate separation of the pathogenic, intermediate, and saprophytic subgroups, and the ability to differentiate 35 currently described Leptospira species with branch supports between 11\% and $100 \%$ (data not shown).

\section{Experimental verification of the PCR-rpoC}

With the PCR assay for $r p o C$, it was possible to amplify the rpoC gene from eight reference strains belonging to pathogenic, intermediate, and saprophytic Leptospira species [Supplementary data (Figure 3-A1)], and the other species were theoretically inferred. Additionally, the usefulness of the PCR assay for detecting Leptospira from human, rodent, and monkey samples naturally infected with Leptospira was verified [Supplementary data (Figure 3-A2)] with an analytical sensitivity of 10 DNA femtograms per sample [Supplementary data (Figure 3-A3)]. Furthermore, we were able to correctly identify the species in human, rodent, and monkey samples by a phylogenetic analysis of the rpoC gene [Supplementary data (Figure 3-B)]. The phylogenetic analysis for the rpoC gene correctly identified the unknown samples at the species level, and these results were $100 \%$ concordant with those obtained by the phylogenetic analysis of the $16 \mathrm{~S}$ ribosomal gene (data not shown). Human, rodent, and monkey sequences were deposited in the NCBI database under the following codes: MK510855, MK510852, MK510856, MK510851, MK510854, MK510853, MK529909, MK529910, MK521701, MK521702, MK521699, and MK521700.

We present a comparative genomic analysis of 35 Leptospira species, finding that the Leptospira species shared approximately 1650 orthologous proteins, and there was a high variability in the amino acid composition of these proteins. These proteins are ideal molecular targets for the implementation of new molecular characterization tools for Leptospira species. In our study, we found approximately 1650 orthologous proteins that were related to vital processes of the bacteria (data not shown). A notable finding was that most of these proteins reflect the molecular speciation process of the Leptospira genus, which may be related to the independent evolutionary process of each subgroup. By taking advantage of this evolutionary process, we propose using the rpoC gene (which encodes the DNA-directed RNA polymerase subunit beta) to implement a phylogenetic identification system that will allow for the identification of unknown isolates at the species level, and to classify them according to their pathogenicity status directly from clinical samples. Additionally, the gene polymorphisms allow for the identification and differentiation of the 35 Leptospira species currently described via the amplification of a 353 bp fragment (amplification of a small fragment helps to improve the sensitivity of PCR assays). The rpoC gene fragment offers better advantages than the initial region of the $16 \mathrm{~S}$ ribosomal gene for species identification, since it can be used to differentiate between L. biflexa/L. wolbachii, L. meyeri/L. macculloughii/L. levettii/L. yanagawae, L. licerasiae/ L. saintgironsiae/L. neocaledonica, L. brenneri/L. harrisiae, and L. venezuelensis/L. haakeii/L. hartskeerlii/L. wolffii.

The initial fragment of the $16 \mathrm{~S}$ ribosomal gene can only discriminate 20 out of the 35 species currently described, while the rpoC gene can be used to identify all 35 species. Another advantage of the rpoC gene is that it can be used to differentiate between intermediate species, which may be present in environmental water sources and soils and can be sources of infection for humans and animals ${ }^{11}$. At the experimental level, the PCR assay for rpoC was successfully used to detect species belonging to pathogenic, intermediate, and saprophytic subgroups. Additionally, the PCR assay for rpoC with unknown samples from humans, rodents, and monkeys was successful in detecting Leptospira with an analytical sensitivity of 10 femtograms of DNA. Furthermore, the phylogenetic analysis for the rpoC gene correctly identified the unknown samples at the species level, and these results were $100 \%$ concordant with those obtained by the phylogenetic analysis of the $16 \mathrm{~S}$ ribosomal gene (data not shown). Therefore, the PCR assay for rpoC could be a valuable tool for the detection and identification of Leptospira species directly from unknown samples with a high analytical sensitivity in addition to the absence of cross-reactions with other bacterial species and uncultivable microorganisms. Currently, the rpoB gene, wzy gene, $S 10$-spc- $\alpha$ locus, and 23S rRNA gene are used to identify Leptospira species, but the 35 species of the Leptospira genus currently described have not been evaluated in these studies ${ }^{12-15}$. In conclusion, this gene could be used as a molecular target in tools to diagnose and identify the main species of the Leptospira genus directly from clinical samples, environmental water sources, and soils.

\section{ACKNOWLEDGEMENTS}

The authors are grateful for the support of CES University, Graduate School, Doctoral Program in Health Sciences, and COLCIENCIAS-Colombia.

\section{AUTHORS' CONTRIBUTION}

RGVJ: The PhD student performed the experimental essays and the writing of the manuscript; PAF: writing and revision of the manuscript; JAL: writing and revision of the manuscript; RGPS: writing and revision of the manuscript. 


\section{CONFLICTS OF INTEREST}

The authors declare that there is no conflict of interest regarding the publication of this paper.

\section{FINANCIAL SUPPORT}

This work was supported by COLCIENCIAS (Project: 122877757660).

\section{REFERENCES}

1. Adler B, de la Peña Moctezuma A. Leptospira and leptospirosis. Vet Microbiol. 2010;140(3-4):287-96.

2. Morey R, Galloway R, Bragg S, Steigerwalt A, Mayer L, Levett PN. Species-specific identification of Leptospiraceae by $16 \mathrm{~S}$ rRNA gene sequencing. J Clin Microbiol. 2006;44(10):3510-6.

3. Backstedt BT, Buyuktanir O, Lindow J, Wunder E, Reis M, UsmanBrown S, et al. Efficient Detection of Pathogenic Leptospires Using 16S Ribosomal RNA. PLoS One. 2015;10(6):e0128913.

4. Fouts D, Matthias M, Adhikarla H, Adler B, Amorim-Santos L, Berg $\mathrm{D}$, et al. What Makes a Bacterial Species Pathogenic?: Comparative Genomic Analysis of the Genus Leptospira. PLoS Negl Trop Dis. 2016;10(2):e0004403.

5. Kumar S, Stecher G, Tamura K. MEGA7: Molecular Evolutionary Genetics Analysis Version 7.0 for Bigger Datasets. Mol Biol Evol. 2016;33(7):1870-4.

6. Li L. OrthoMCL: Identification of Ortholog Groups for Eukaryotic Genomes. Genome Res. 2003;13(9):2178-89.
7. Wang Y, Coleman-Derr D, Chen G, Gu Y. OrthoVenn: a web server for genome wide comparison and annotation of orthologous clusters across multiple species. Nucleic Acids Res. 2015;43(W1):W78-W84.

8. Ostlund G, Schmitt T, Forslund K, Kostler T, Messina D, Roopra S, et al. InParanoid 7: new algorithms and tools for eukaryotic orthology analysis. Nucleic Acids Res. 2009;38(Database):D196-D203.

9. Aziz R, Bartels D, Best A, DeJongh M, Disz T, Edwards R, et al. The RAST Server: Rapid Annotations using Subsystems Technology. BMC Genomics. 2008;9(1):75.

10. Thibeaux R, Girault D, Bierque E, Soupe-Gilbert M, Rettinger A, Douyere A, et al. Biodiversity of Environmental Leptospira: Improving Identification and Revisiting the Diagnosis. Front Microbiol. 2018;9:816.

11. Chiriboga J, Barragan V, Arroyo G, Sosa A, Birdsell D, España $\mathrm{K}$, et al. High Prevalence of Intermediate Leptospira spp. DNA in Febrile Humans from Urban and Rural Ecuador. Emerg Infect Dis. 2015;21(12):2141-7.

12. Chandan S, Sharanaiah U, Govindaraju S, Vinayaka B. Use of rpoB gene analysis for detection and identification of Leptospira species by direct sequencing. European J Biotechnol Biosci. 2016;4(1):34-43. .

13. Wangroongsarb P, Chanket T, Gunlabun K, Long D, Satheanmethakul $\mathrm{P}$, Jetanadee $\mathrm{S}$, et al. Molecular typing of Leptospira spp. based on putative O-antigen polymerase gene (wzy), the benefit over 16S rRNA gene sequence. FEMS Microbiol Lett. 2007;271(2):170-9.

14. Victoria B, Ahmed A, Zuerner R, Ahmed N, Bulach D, Quinteiro J, et al. Conservation of the S10-spc- $\alpha$ Locus within Otherwise Highly Plastic Genomes Provides Phylogenetic Insight into the Genus Leptospira. PLoS ONE. 2008;3(7): e2752.

15. Sasaki F, Kano R, Nakamura Y, Tsujimoto H, Yamamoto S, Hasegawa A. Phylogenetic analysis of Leptospira strains of pathogenic serovars using 23S rDNA gene sequences. Microbiol Res. 1999;154(2):167-72. 